\title{
Entwicklungen und Strukturen des ehrenamt- LICHEN ENGAGEMENTS FÜR GEFLÜCHTETE SEIT 2015. Vier BeISPIELE AUS ÖSTERREICHISCHEN REGIONEN
}

\author{
Hannah LichtenWagner, Sonja Nechansky, Sandra Punz, Andreas \\ SCHWARZBAUER, MA-Studium Kultur- und Sozialanthro- \\ pologie, Universität Wien
}

\section{INHALT}

1 Zivilgesellschaftliches Engagement und die Flüchtlingsbewegung von 2015/16_....266

1.1 Die Integration von Geflüchteten in Österreich im zeitlichen Verlauf.............267

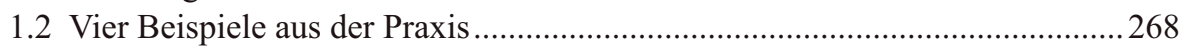

2 Die drei Gemeinden im Mostviertel: Lokaler Kontext der empirischen Erhebung..... 268

2.1 Unterschiedliche Strukturen ehrenamtlichen Engagements in

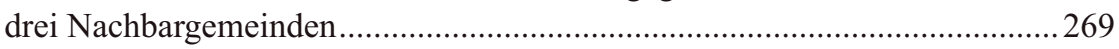

2.2 Ehrenamtliches Engagement und emotionale Dimensionen von Integration....271

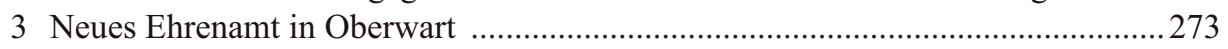

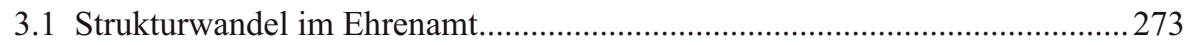

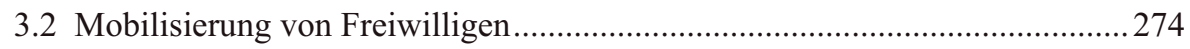

3.3 Sinkendes Engagement........................................................................... 275

4 St. Andrä-Wördern: lokaler Kontext der empirischen Erhebung ............................2278

4.1 Arbeitsteilung auf lokaler Ebene in St. Andrä-Wördern ...................................228

4.2 Politische Dimensionen des Engagements von Freiwilligen ...........................221

5 Politische Strukturen zur Förderung der Integration in Oberösterreich ...................283

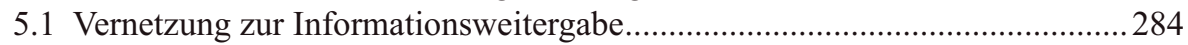

5.2 Strukturierung des freiwilligen Engagements vor Ort - ReKIs .......................228

5.3 „Zusammen Helfen in OÖ“ - Die Rolle der Zivilgesellschaft bei der Integration.287

6 Dimensionen ehrenamtlichen Engagements für Geflüchtete .................................228

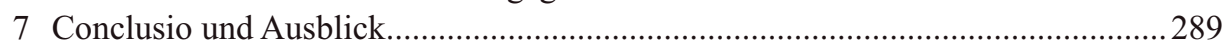

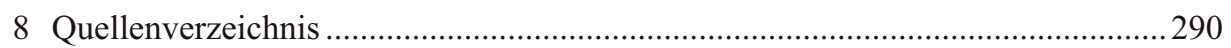

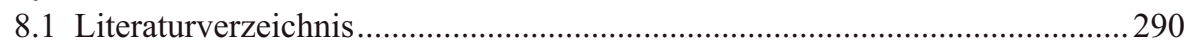

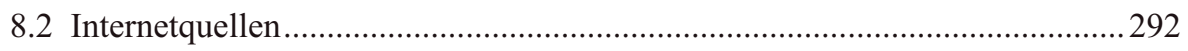

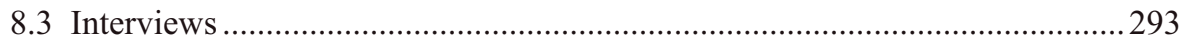




\section{Zivilgesellschaftliches Engagement und die Flüchtlings- bewegung von 2015/16}

Die Jahre 2015 und 2016 stellen mit Sicherheit einen entscheidenden Wendepunkt des zivilgesellschaftlichen Engagements für Geflüchtete in Österreich dar. ${ }^{1}$ Dies bezieht sich sowohl auf die Anzahl der Geflüchteten in Österreich als auch auf den Umfang, in welchem sich Freiwillige für diese engagiert haben. In diesen beiden Jahren wurden in Österreich ungefähr 130.000 Asylanträge gestellt, ${ }^{2}$ was die staatlichen Strukturen zur Aufnahme von Geflüchteten vor große Herausforderungen stellte. Ausgehend von einer sichtbaren Überforderung der öffentlichen Stellen mit der Situation auf menschenwürdige Weise umzugehen, entstanden vielerorts in Österreich zivilgesellschaftliche Initiativen zur Unterstützung von Geflüchteten (SimSA 2017, p. 87). Die Entwicklungen seither können aus unterschiedlichen Gründen als bedeutende Zäsur in diesem Bereich eingestuft werden. Pries (2018, p. 4-7) identifiziert auf europäischer Ebene mehrere Faktoren, welche die Ereignisse in diesem Zeitraum charakterisieren. Der Fokus dieses Artikels liegt auf dem Beitrag, den zivilgesellschaftliche Akteur/ inn/e/n in verschiedenen österreichischen Gemeinden und Regionen in Bezug auf die Integration geflüchteter Menschen leisten. Nach Fleischmann und SteinhilPer (2017, p. 19) hatte die Einordnung der Geschehnisse als „Flüchtlingskrise“ unter anderem auch einen mobilisierenden Effekt, durch welchen Ehrenamtliche zu humanitärer Hilfe für Geflüchteten in den ersten Wochen und Monaten nach deren Ankunft motiviert wurden. Seit dem Jahr 2015 lässt sich außerdem eine Diversifikation der Akteur/innen in diesem Bereich feststellen. So engagierten sich neben spontan entstehenden Initiativen von Ehrenamtlichen auch bereits etablierte soziale Bewegungen und NGOs, migrantische Gruppierungen und Unternehmen mit Spenden und finanzieller Unterstützung (CANTAT \& FeIschmidT 2018, p. 384 f.). An dieser Stelle ist zu betonen, dass es sich im Falle der Zivilgesellschaft nicht um eine homogene Gruppe von Akteur/inn/e/n handelt (FIORAMONTI \& THÜMLER 2013) und die einzelnen Ehrenamtlichen durchaus divergierende Ansichten zu den Themen Flucht und Integration vertreten. Trotzdem lässt sich ein gemeinsamer Nenner im praktischen Engagement für Geflüchtete nachweisen.

\footnotetext{
Die in diesem Beitrag präsentierten Ergebnisse wurden von den Autor/inn/en im Rahmen der jeweiligen Masterarbeit erhoben. Diese Abschlussarbeiten sind Teil des Forschungsprojektes „Loslassen-Durchstehen-Ankommen“ (LODA) der Österreichischen Akademie der Wissenschaften und wurden aus diesem auch finanziell unterstützt. Der Zeitraum der Datenerhebung erstreckte sich vom Frühling bis zm Winter 2018.

$2 \quad 88.340 \mathrm{im}$ Jahr 2015 und $42.285 \mathrm{im}$ Jahr 2016; im Vergleich dazu waren es 2014 nur 28.064 (STATISTIK Austria 2018: <http://www.statistik.at/wcm/idc/idcplg?IdcService=GET_PDF_FILE $\&$ RevisionSelectionMethod=LatestReleased\&dDocName=022914 $>$ [Zugriff am 10.01.2019].
} 


\subsection{Die Integration von Geflüchteten in Österreich im zeitlichen Verlauf}

Für die Integration von Geflüchteten generell und so auch in Österreich spielt vor allem der Faktor Zeit eine wichtige Rolle. SIMSA (2017, pp. 84-86) unterscheidet im „Life Cycle of Refugee Integration“ im Hinblick auf die zeitliche Dimension drei verschiedene Abschnitte: unmittelbare Bedürfnisse von Geflüchteten nach der Ankunft in Österreich, die Situation während des Asylverfahrens und die Zeit nach dem Erhalt eines Aufenthaltstitels in Österreich. In der ersten Phase geht es vor allem darum, basale Bedürfnisse wie Wohnraum, Kleidung, Verpflegung und medizinische Versorgung sicherzustellen. Hierbei spielten vor allem Ende 2015 und Anfang 2016 zivilgesellschaftliche Akteur/ inn/e/n eine entscheidende Rolle. Nach der Zulassung zum Asylverfahren in Österreich haben Geflüchtete zwar nach wie vor Anspruch auf grundlegende Versorgung, allerdings dauern die Verfahren in vielen Fällen so lange, dass mit der Zeit darüber hinaus auch andere Themen zusätzlich an Bedeutung erlangen. Asylwerber/innen sind in Österreich bis auf wenige Ausnahmen vom regulären Arbeitsmarkt ausgeschlossen ${ }^{3}$ (Bartenstein Erlass 20044) und verfügen daher über geringe finanzielle Mittel, die sie selbstständig verwalten können. Daraus folgt, dass Sprachkurse meist nur besucht werden können, wenn diese kostenlos angeboten werden. Dazu kommt vor allem im ländlichen Raum das Problem der Finanzierung von Fahrkarten, da die Kurse häufig nicht in unmittelbarer Nähe der Unterkünfte stattfinden. Außerdem ist die Situation von Asylwerber/ inne/n von großer Unsicherheit gekennzeichnet, was den Ausgang des Verfahrens betrifft (LANGTHALER \& TRAUNER 2009, p. 462), und von langen Warteperioden geprägt. Nach dem positiven Abschluss des Asylverfahrens verändert sich die Situation von Geflüchteten in Österreich schlagartig. Auf der einen Seite haben sie Anspruch auf Deutschkurse und Sozialleistungen wie die bedarfsorientierte Mindestsicherung sowie uneingeschränkten Zugang zum Arbeitsmarkt. Andererseits stehen viele Asylberechtigte dann vor der Herausforderung innerhalb kurzer Zeit eine geeignete Unterkunft zu finden. Auch in dieser Phase leisten Ehrenamtliche wichtige Unterstützung für Asylberechtigte bei der Suche nach Bildungsangeboten, Wohnraum und Arbeit.

Die hier beschriebenen Phasen des „Life Cycle of Refugee Integration“ und die damit verbundenen Bedürfnisse von Geflüchteten haben auch Auswirkungen auf die Schwerpunkte der Unterstützung von Ehrenamtlichen. Im Laufe der Zeit treten Sofortmaßnahmen und humanitäre Hilfe in den Hintergrund und Maßnahmen zur strukturellen Integration wie Sprachkurse, andere Bildungsangebote und Unterstützung bei der Arbeitssuche werden wichtiger. Während dieser drei Abschnitte sind ehrenamtliche Unterstützer/innen für Geflüchtete wichtige Ansprechpersonen und tragen durch ihr Engagement und den Aufbau persönlicher Beziehungen dazu bei, dass Geflüchtete auch auf einer emotionalen, zwischenmenschlichen Ebene in Österreich ankommen können.

\footnotetext{
Dazu gehören unter bestimmten Auflagen gemeinnützige Tätigkeiten, Saisonarbeit und Arbeitsplätze im Tourismusbereich.

${ }_{4}$ UNDOK 2018: <http://arbeitsmarktzugang.undok.at/2013/02/06/faq-4/> [Zugriff am 12.01.2019]
} 


\subsection{Vier Beispiele aus der Praxis}

Der weitere Inhalt dieses Beitrags unterteilt sich in vier Abschnitte, die sich mit zivilgesellschaftlichem Engagement für Geflüchtete in verschiedenen Regionen in Österreich seit den Jahren 2015/16 beschäftigen. Es gilt zu beachten, dass sich die Rahmenbedingungen seit dieser Zeit maßgeblich verändert haben. In den letzten beiden Jahren sind vergleichsweise wenige Geflüchtete nach Österreich gekommen und die politischen Machtverhältnisse sowie der öffentliche Diskurs in Österreich haben sich ebenfalls gewandelt. Seit der Nationalratswahl 2017 wird die Regierung von einer Koalition aus ÖVP und FPÖ gebildet, die einen restriktiveren Kurs Geflüchteten gegenüber eingeschlagen hat. Von der „Willkommenskultur“ der ersten Wochen und Monate während der so genannten „refugee crisis“ ist nicht mehr allzu viel übriggeblieben, Flucht und Integration werden derzeit vor allem als Sicherheitsthema diskutiert (SCHEIBELHOFER 2017). Auf Grund dieser Entwicklungen werden in vielen Städten und Gemeinden Österreichs Unterkünfte für Geflüchtete geschlossen, Gelder für Integrationsmaßnahmen gekürzt und Förderprogramme gestrichen (KAPELLER 2018). Diese geänderten Bedingungen bilden den Rahmen, in welchem zivilgesellschaftliches Engagement für Geflüchtete in Österreich derzeit stattfindet.

Die empirischen Forschungen zu diesem Beitrag wurden einerseits in drei unterschiedlichen, eher ländlichen Regionen Österreichs, andererseits im Bundesland Oberösterreich durchgeführt. Im ersten Teil werden drei Gemeinden im Mostviertel verglichen und deren unterschiedliche Strukturen ehrenamtlichen Engagements herausgearbeitet. Im Zentrum der Analyse stehen dabei verschiedene Beziehungsformen zwischen Geflüchteten und Ehrenamtlichen und deren Bedeutung für die lokale Integration. Der zweite Teil beschäftigt sich mit der Region Oberwart im Südburgenland und den dortigen Herausforderungen für ehrenamtliches Engagement. Mehrere Kausalfaktoren, weshalb zivilgesellschaftliche Aktivitäten hier seit 2015/16 signifikant abgenommen haben, werden analysiert. Im dritten Teil werden die verschiedenen Akteur/inn/e/n beschrieben, die sich mit der lokalen Integration in der Gemeinde St. Andrä-Wördern (NÖ) befassen. Dabei stehen vor allem Unterschiede in der Zugangsweise der Gemeinde einerseits und von ehrenamtlichen Initiativen andererseits sowie deren politische Dimensionen im Mittelpunkt. Den Abschluss bildet der vierte Teil, in welchem die seit 2015 entwickelten Strukturen zur Integration von Geflüchteten und Unterstützung von Ehrenamtlichen in Oberösterreich behandelt werden.

\section{Die drei Gemeinden im Mostviertel: Lokaler Kontext der empirischen Erhebung}

Das erste empirische Beispiel befasst sich mit drei Gemeinden (A, B und C) im niederösterreichischen Mostviertel. Die Region ist in rund 1,5 Stunden mit dem Auto 
von Wien erreichbar, eine Nebenbahnstrecke verbindet die Gemeinden im Stundentakt mit St. Pölten. Die drei Gemeinden weisen ähnliche politische, wirtschaftliche und soziale Rahmenbedingungen auf. Mit ca. 70 Prozent ist jeweils die lokale ÖVP-nahe Liste $^{5}$ am stärksten im Gemeinderat vertreten, gefolgt von SPÖ und FPÖ. Von der erwerbstätigen Gemeindebevölkerung arbeitet nur ein Drittel bis ein Viertel in der Nähe des Wohnortes, die überwiegende Mehrheit der Bevölkerung pendelt zum Arbeiten aus. ${ }^{6}$ Alle drei Gemeinden weisen eine belebte Vereinslandschaft auf.

Trotz der großen Ähnlichkeiten in Bezug auf politische, wirtschaftliche und soziale Rahmenbedingungen der drei Nachbargemeinden manifestieren sich doch deutliche Unterschiede, wenn es um die Erfahrungen mit Geflüchteten geht. Bereits in den 1980er Jahren fanden Menschen aus den ehemaligen Ostblockstaaten sowie dem ehemaligen Jugoslawien in den Orten zeitweilig Zuflucht. Diese historische Entwicklung betraf vor allem Gemeinde A und B. Ähnlich wie auch bei der rezenten Fluchtbewegung zu beobachten, siedelten sich kaum geflüchtete Menschen dauerhaft an. Gemeinde B hat seither mit kurzen Unterbrechungen laufend Asylwerber/innen in Grundversorgungsquartieren untergebracht. In Gemeinde A und C haben seit dem Spätsommer 2015 wieder zahlreiche Geflüchtete aus den Ländern Afghanistan, Irak, Iran, Syrien und Tschetschenien einen vorübergehenden Wohnort gefunden.

\subsection{Unterschiedliche Strukturen ehrenamtlichen Engagements in drei Nachbargemeinden}

Die lokalen Strukturen zivilgesellschaftlichen Engagements gestalten sich in den drei Gemeinden sehr spezifisch. Während in Gemeinde A mit der Aufnahme von Geflüchteten ein Verein gegründet wurde, welcher sich der lokalen Unterstützung von Geflüchteten widmet, wird ehrenamtliches Engagement in Gemeinde C hauptsächlich durch Einzelpersonen geleistet. Wieder anders stellt sich die Situation in Gemeinde B dar, welche in Bezug auf die Fluchtmigration auf langjährige historische Erfahrungen zurückgreifen kann. Zivilgesellschaftliche Tätigkeiten für Geflüchtete konzentrieren sich vor allem auf den Bereich der Pflichtschule.

In Gemeinde A wurde im Herbst 2015 ein Verein ins Leben gerufen, um die vorhandene breite Unterstützungsbereitschaft für Geflüchtete durch die Zivilgesellschaft besser koordinieren zu können. Neben der finanziellen Unterstützung in diversen Belangen (es wurde ein Spendenkonto eingerichtet) und einem Sachspendenlager stellte vor allem der kostenlose Deutschunterricht eine wesentliche Säule dar, welcher über einen Zeitraum von drei Jahren von 30 bis 40 Ehrenamtlichen geleistet wurde. Der

\footnotetext{
Für die Parteien ist immer eine Kombination aus Ortsname und der Bezeichnung ,(Österreichische) Volkspartei“ namensgebend, welche das Naheverhältnis der Ortslisten zur ÖVP kennzeichnet.

6 GemeindestatistiK Niederösterreich: < <http:/www.noe.gv.at/noe/St-Poelten/Gemeinden im_Bezirk_St._Poelten.html $>$ [Zugriff am 08.01.2019]
} 
Verein wurde im Mai 2018 aufgelöst, da der Bedarf nicht mehr gegeben war. Dafür waren mehrere Gründe relevant, die alle drei Gemeinden gleichermaßen betreffen: Zum einen hat sich gezeigt, dass sich geflüchtete Menschen nach Erhalt eines positiven Asylbescheids nicht langfristig in den Gemeinden ansiedeln. Als Asylberechtigte ziehen sie aus unterschiedlichen Gründen in größere Städte wie St. Pölten und sehr häufig nach Wien. Doch auch im umgekehrten Fall, wenn Asylwerber/innen eine zeitnahe Abschiebung befürchten oder tatsächlich abgeschoben werden, verlassen sie die Orte plötzlich. Zum anderen liegt die gesunkene Nachfrage auch daran, dass aktuell nur wenige Asylwerber/innen neu in die Gemeinde kommen.

Einmal aufgebaute Beziehungen zwischen Geflüchteten und Personen, die sich über den Verein engagiert haben, bleiben zum Teil nach der Auflösung des Vereins weiterhin bestehen. Selbst wenn kein Deutschunterricht mehr stattfindet, verlagert sich das ehrenamtliche Engagement mitunter vom Rot-Kreuz-Haus ins private Wohnzimmer. Astrid, ${ }^{7}$ eine pensionierte Lehrerin aus der Gemeinde A, engagiert sich seit zwei Jahren mit ihrem Ehemann im Ort. Astrid schilderte in einem Interview, wie sich ihr Engagement seit der Auflösung des Vereins verändert hat. Dabei kümmert sie sich im Speziellen um Arman, einen jungen, alleinstehenden Geflüchteten aus Afghanistan.

„Nachdem ich begonnen habe sie zu unterrichten, haben sich da menschliche Verbindungen aufgebaut und ja - jetzt besuchen sie mich alle ab und zu noch. [...] Ja, und wenn es Probleme gibt und so, dann kommen sie halt zu mir und fragen. Natürlich auch finanziell. [...] Arman ist [...] unser Hauptkind. [...] Also wir lernen mit Arman, wir hören uns seine Probleme an. [...] Wir verfassen sogar - oder sagen ihm, was er schreiben kann-SMS an seine neu erworbene Freundin. Also all diese Probleme, die ein 19-Jähriger eben hat. Oder wir erklären ihm [die] Feiertage und kaufen ihm [einen] Taschenrechner. [...] Oder er hat einen Computer von uns bekommen. " (Interview mit Astrid, Ehrenamtliche Gemeinde A, 15.11.2018)

An diesem Beispiel wird die ganze Bandbreite ehrenamtlichen Engagements deutlich: Dieses reicht von finanzieller Hilfe und Sachspenden über Lernbegleitung bis hin zu emotionaler Unterstützung. Die Freiwillige übernimmt in diesem Sinne auch eine Elternrolle, wie Arman selbst betont.

In der Gemeinde B haben insbesondere die Direktor/inn/en von Volks- und Neuer Mittelschule langjährige Erfahrungen mit der Integration von geflüchteten Kindern und Jugendlichen. Das hängt auch damit zusammen, dass eines der beiden Grundversorgungsquartiere ausschließlich Familien aufnimmt. Vor fünf Jahren wurde daher das „Senior/inn/en-Lehrer/innen-Projekt“ ins Leben gerufen, wobei eine Gruppe von zehn Pensionist/inn/en täglich zwei mal zwei Unterrichtsstunden abhalten, in welchen sie mit den Kindern Deutsch lernen und bei Aufgaben helfen. Dabei sind die Senior/inn/en gut in die professionellen Strukturen der Schule eingebunden. Die Lehrer/innen bereiten Arbeitsmaterial vor, welches die geflüchteten Kinder gemeinsam mit den Senior/inn/

Alle angeführten Namen wurden anonymisiert. 
en bearbeiten. Bleibt noch etwas Zeit übrig, werden Spiele gespielt. Die Senior/inn/ en-Lehrer/innen nehmen im Laufe der Zeit für manche Kinder eine Großelternrolle ein. Dies wird im folgenden Zitat deutlich:

„Brigitte: Naja, wir sind ein bisschen Omas.

Bruno: Ja, ist eh klar.

Beate: Na sicher.

Brigitte: Oder Opas, für die Kinder. Also ...

Beate: $\quad$ Ich meine, es ist ja dann schön, wenn sich dann ein Kind einmal - am Anfang natürlich sind sie ein bisschen zurückhaltend, aber wenn sich dann einmal ein Kind so richtig ...

Brigitte: Naja, öffnet.

Beate: Öffnet-ja, für uns.

Bruno: Die kommen privat auch auf uns zu. [...]

Beate: Das war ja auch lieb. Hat mir eine Mutter zum Muttertag - ich meine mir, eine Mutter zum Muttertag - einen riesen Blumenstock gebracht. " (Gruppeninterview mit drei Senior/inn/en-Lehrer/innen, 24.10.2018)

Die Geste der Mutter eines geflüchteten Kindes ist in diesem Zusammenhang bemerkenswert aufgrund ihres symbolischen Charakters, weil damit die Beziehung zwischen der Ehrenamtlichen und dem Kind ausgedrückt wird.

Dass die in der Gemeinde C wohnhaften Geflüchteten seit 2015 ehrenamtliche Unterstützung erhalten, ist der Vernetzungsarbeit der Diakonie zu verdanken. Da es schwierig war, eine Person aus der Region für einen kostenlosen Deutschkurs zu gewinnen, reiste von Herbst 2015 bis Anfang 2018 einmal wöchentlich Claudia, eine Freiwillige aus Wien, in die Gemeinde, um mit den Geflüchteten integrativ-pädagogisch zu arbeiten. Dabei stand zwar das Deutschlernen im Mittelpunkt, für Claudia ist es jedoch genauso wichtig, Kontakte zur lokalen Bevölkerung zu knüpfen. Hierzu vermittelte sie die Geflüchteten in Vereine und ermutigte sie zur Beteiligung an ehrenamtlicher Tätigkeit. Für die Geflüchteten ist sie eine der wenigen Kontaktpersonen zur österreichischen Bevölkerung, eine Brückenbauerin. Ihre Arbeit bleibt nicht auf Gemeinde C beschränkt, sondern erweitert sich im Falle eines Umzugs nach Wien nach Erhalt eines positiven Asylbescheids auf Hilfeleistungen bei der Wohnungs- und Arbeitssuche.

\subsection{Ehrenamtliches Engagement und emotionale Dimensionen von Integration}

Der Alltag der meisten Geflüchteten, die in den drei Gemeinden wohnen, ist gekennzeichnet durch Warten. Viele haben bereits einen negativen Bescheid erhalten 
und warten seit Monaten, manche seit über einem Jahr, auf ein zweites Interview. Im Sinne von Simsa (2017, pp. 84 ff.) befinden sich die Geflüchteten in der zweiten Phase des „Life Cycle of Refugee Integration“.

Die Ehrenamtlichen in der Region sind in dieser Phase des Wartens für die Geflüchteten wichtige Kontaktpersonen zur österreichischen Bevölkerung und tragen somit wesentlich zur seelisch-emotionalen Integration bei. HAN-BROICH (2012, pp. 115 ff.) hat diesbezüglich drei Dimensionen von Integration definiert. ${ }^{8}$ Bei der seelisch-emotionalen Integration geht es darum, dass sich die Migrant/inn/en als Menschen akzeptiert, respektiert und willkommen fühlen. Bei der Integration auf der kognitiv-kulturellen Ebene steht der Spracherwerb im Vordergrund, jedoch fällt auch die Kenntnis von Normen und Werten der Aufnahmegesellschaft unter diese Kategorie. Integration auf der sozial-strukturellen Ebene bedeutet, dass Migrant/inn/en Zugang zu Vereinen, dem Arbeits- und Wohnungsmarkt, dem Bildungssektor und schließlich zu staatsbürgerlichen Rechten haben. Dabei sind die Dimensionen weder als zeitliche Abfolge noch voneinander getrennt zu verstehen. Positive Integrationserfahrungen auf einer Ebene wirken sich auch positiv auf eine andere aus (HAN-BRoICH 2012, p. 147). Die ehrenamtliche Arbeit mit Geflüchteten (als eine spezifische Gruppe von Migrant/inn/en) leistet insbesondere auf der seelisch-emotionalen Ebene einen entscheidenden Beitrag und ebnet damit den Weg für die kognitiv-kulturelle und sozial-strukturelle Dimension von Integration (HAN-BROICH 2012, p. 184).

In ähnlicher Weise argumentiert SIMSA (2012, p. 84), dass zwischen „,functional and affectional integration" unterschieden werden muss. Erstere bezieht sich auf Unterstützungsleistungen im formalen Bildungs-, Arbeits- und Wohnungssektor sowie bei juristischen Belangen. „Affectional integration“ wird ihr zufolge durch gemeinsame regelmäßige Tätigkeiten gefördert, wobei „relationship work“ stattfindet, also Beziehungen zwischen Geflüchteten und Ehrenamtlichen entstehen. SimSA zufolge sind Ehrenamtliche zwar in beiden Bereichen tätig, den bedeutsameren Beitrag leisten Ehrenamtliche allerdings auf dem Gebiet der ,,affectional integration“. Anders als bei HAN-BROICH werden Aktivitäten, die z. B. auf die Vermittlung von Sprache oder Verhaltensnormen der Aufnahmegesellschaft abzielen, nicht von der emotional konnotierten Kategorie von Integration abgetrennt betrachtet. Vielmehr kommt es auf die Qualität der Interaktion an, d. h., ob die Interaktion zum Aufbau von Beziehungen führt oder nicht.

Laut HAN-Broich (2012, pp. 157 ff.) lassen sich Beziehungen zwischen Ehrenamtlichen und Geflüchteten ihrer Funktion nach in vier Typen einteilen: Ersatz-, Kompensations- ${ }^{9}$, Lern- und Kapitalbeziehung. Die oben dargestellte Großelternrolle der Senior/

\footnotetext{
8 Han-BroIch hat ein Indikatorenmodell entwickelt, mit welchem Integration messbar gemacht werden soll. Das Indikatorenmodell wurde in dieser Fallstudie nicht als Erhebungsinstrument verwendet, die von Han-Broich definierten Dimensionen werden lediglich für die Analyse genutzt.

9 Inwieweit die Ehrenamtlichen in der Region mit den Geflüchteten eine Kompensationsbeziehung eingehen, konnte nicht festgestellt werden. Die Kompensationsbeziehung wird von HAN-BROICH als Beziehung definiert, in welcher Geflüchtete positive Erfahrungen mit Menschen der Aufnahmegesellschaft machen und somit negative Erfahrungen mit z. B. Mitarbeiter/inne/n
} 
inn/en-Lehrer/innen fällt ebenso unter die Kategorie der Ersatzbeziehung wie die Elternrolle, welche Astrid für Arman übernimmt. Allerdings gehen die Ehrenamtlichen parallel auch eine Lernbeziehung mit den Geflüchteten ein. Dabei steht zwar die Sprachvermittlung im Vordergrund, der Lerneffekt ist jedoch nicht einseitig zu verstehen. Auch die Ehrenamtlichen lernen durch die Interaktionen mit den Geflüchteten dazu. Claudias Engagement kann im Beziehungsmodell HAN-BROICHs sowohl als Lern- als auch als Kapitalbeziehung betrachtet werden. Eine Kapitalbeziehung von Geflüchteten zu Ehrenamtlichen kann ,,in bestimmten Situationen, bspw. bei behördlichen Schwierigkeiten oder bei der Wohnungs- und Arbeitssuche, nutzbringend und vorteilhaft für Flüchtlinge“"(HAN-BROICH 2012, p. 165) sein.

Wie die empirischen Befunde der Gemeinden A, B und C zeigen, setzt ehrenamtliche Integrationsarbeit bei der seelisch-emotionalen und kognitiv-kulturellen Dimension bzw. der ,affectional integration“ an. Für Geflüchtete ist es wichtig, Möglichkeiten zu erhalten mit Menschen der Aufnahmegesellschaft in Kontakt zu treten und positive Erfahrungen zu machen, als Menschen akzeptiert und willkommen zu sein. Ehrenamtliche leisten in diesem Bereich einen unverzichtbaren Beitrag zur Integration. Auf die Herausforderungen, die ehrenamtliches Engagement für Geflüchtete mit sich bringt, wird im folgenden Abschnitt genauer eingegangen.

\section{Neues Ehrenamt in Oberwart}

Dieser Abschnitt thematisiert die Freiwilligenarbeit für Geflüchtete im Bezirk Oberwart im Südburgenland. Es wird untersucht, wie sich das Engagement der Freiwilligen seit dem Spätsommer 2015 entwickelt hat und inwiefern auch in Oberwart ein Strukturwandel im Ehrenamt zu erkennen ist, der in den vergangenen zwei Jahrzehnten in der wissenschaftlichen Literatur immer wieder Erwähnung fand. Ehrenamtlich Tätige würden sich demnach weniger in traditionellen Vereinen organisieren, sondern ihre Tätigkeit vermehrt selbst gestalten (HAN-BROICH 2012, p. 78).

\subsection{Strukturwandel im Ehrenamt}

Seit den 2000er-Jahren beschäftigen sich Wissenschafter/innen vermehrt mit strukturellen Veränderungen im Ehrenamt. Das traditionelle Ehrenamt, das zum Beispiel durch die Freiwillige Feuerwehr, durch Sportvereine oder durch Kirchen organisiert ist, wurde um eine neue Variante des Ehrenamts ergänzt. Neben dem langjährigen und kontinuierlichen Engagement hat sich eine Form entwickelt, welche als „kurzfristig,

staatlicher Behörden ausgeglichen werden können bzw. erfahren sie durch den Kontakt zu Ehrenamtlichen eine persönliche Aufwertung ihres eigenen sozialen Status. 
lebensnah, themenspezifisch und wechselhaft" beschrieben wird (HAN-BROICH 2012, p. 78).

Viele Freiwillige wollen sich nicht mehr durch gesellschaftliche Traditionen und Institutionen leiten lassen, sondern organisieren und gestalten ihre Tätigkeit selbst (JANOwicz et al. 2000, p. 19). So schreibt auch RudolPh, dass Personen, die sich für diese Art des Ehrenamts entscheiden, die „Unterordnung unter die Strukturen eines Wohlfahrtsverbands oder einer kirchlichen Organisation" ablehnen (RUDOLPH 2003, p. 126). Generell zeige sich daher ein Rückgang der Zahl, der im Rahmen von Verbänden oder in kirchlich organisierten Ehrenamtsbereichen Engagierten. Analoges gilt für Vereine und politische Parteien (ebd.).

Auch Han-Broich thematisiert in ihrem Buch „Ehrenamt und Integration“ den Strukturwandel im Ehrenamt. Die heutige Tendenz geht ,hin zu Bereichen und Vereinigungen kollektiv organisierter Selbst- und Fremdhilfe (Sport-, Freizeit- und Kulturbereich, Bürgerinitiativen sowie Selbsthilfegruppen), in denen man sich flexibel, projektorientiert und zeitlich begrenzt engagieren kann" (HAN-BROICH 2012, p. 78).

Mit dem Strukturwandel geht auch eine Veränderung der Motive einher. Während früher altruistische und karitative Motive im Mittelpunkt gestanden sind, werden in neueren Studien immer wieder individualistische und egoistische Motive für ehrenamtliches Engagement festgestellt (vgl. KÜHNLEIN \& BöHLE 2002; ZimMER \& VILAIN 2005; REINERT 2005). „Man will Spaß haben, eigene Fähigkeiten und Kenntnisse einbringen und weiterentwickeln. Man will mit interessanten Leuten gemeinsam etwas bewegen und durch das Engagement gesellschaftlich partizipieren“, thematisiert auch RuDOLPH die veränderten Motivationen (RUDOLPH 2003, p. 126). Wichtig ist es aber auch festzuhalten, dass traditionelle Motive nicht unbedingt abnehmen müssen: Rudolph geht davon aus, dass es eher zu einem „Motivmix“ kommt, denn „,an ehrenamtlichen Tätigkeit ,Spaß haben' und ,anderen helfen' muss sich ja nicht notwendigerweise ausschließen“ (ebd.). KüHNLEIN \& BöHLE betonen jedoch, dass Formen des ehrenamtlichen Engagements, die auch die persönlichen Bedürfnisse von Ehrenamtlichen treffen, an Bedeutung gewinnen werden (2002).

Im Folgenden soll analysiert werden, ob diese neue Form des Ehrenamts seit dem Spätsommer 2015 auch in Oberwart zu beobachten ist.

\subsection{Mobilisierung von Freiwilligen}

In Oberwart kam es im September 2015 wie auch in vielen anderen Orten in Österreich zu einer intensiven Mobilisierung von Freiwilligen, die sich für Geflüchtete einsetzten. Die Nähe zur ungarischen Grenze veranlasste viele Oberwarter/innen, Sachspenden (z.B. nach Nickelsdorf) zu bringen. Später unterstützten zahlreiche Freiwillige die Versorgung der mehr als 1.500 Geflüchteten, die in der Oberwarter Messehalle untergebracht waren. 
Anschließend kam es zu einer Aufstockung der Grundversorgungsquartiere im Bezirk, und es wurden Vereine wie „Flüchtlingsinitiative Südburgenland“ oder „Begegnung in Pinkafeld“ gegründet, die sich mit den Anliegen und Bedürfnissen der Geflüchteten beschäftigten sowie Sprachcafés, Nähtreffs und Ähnliches organisierten. Der Flüchtlingsdienst der Diakonie hat den Auftrag des Landes erhalten, sich um die Regionalbetreuung zu kümmern und ist in dieser Zeit stark gewachsen. Es wurde auch ein Forum gegründet, in dem das Zusammentreffen der lokalen Bevölkerung und der Geflüchteten durch das Abhalten von Workshops etc. aktiv gefördert wird.

\subsection{Sinkendes Engagement}

Zum Zeitpunkt der Feldforschung waren ca. drei Jahre seit der Aufnahme der Geflüchteten im Spätsommer 2015 vergangen und viele der zivilgesellschaftlichen Projekte oder Vereine, die in dieser Zeit gegründet wurden, waren mittlerweile geschrumpft oder nicht mehr aktiv. Derzeit gibt es in Oberwart eher einzelne Personen, die sich freiwillig mit den Anliegen und Bedürfnissen der Geflüchteten beschäftigen.

In den Interviews werden verschiedene Gründe für Demotivation genannt. So wird beispielsweise das Fehlen von finanziellen Ressourcen mehrfach betont. Interviewpartner/innen halten fest, dass sie alle Kosten wie z.B. für Kopien selbst tragen mussten. Diese fehlenden Ressourcen werden auch hinsichtlich der Mobilität deutlich. Denn die meisten Angebote für Geflüchtete finden und fanden in der Stadtgemeinde Oberwart statt, doch ein Großteil der Grundversorgungsquartiere liegt außerhalb. Weil Bustickets $\mathrm{zu}$ teuer sind und auch regelmäßige öffentliche Verbindungen nicht besonders ausgebaut sind, müssen bzw. müssten Geflüchtete mehrere Kilometer zu Fuß zurücklegen, um die freiwilligen Angebote der Diakonie oder von der Flüchtlingsinitiative in Anspruch nehmen zu können.

Generell wurde von den Freiwilligen auch ein Rechtsruck in der Bevölkerung beschrieben und sie berichten von politischen Konflikten, die ihre Tätigkeit hervorruft:

„,...] für mich ist Helfen eine Selbstverständlichkeit. Ich bin damit irgendwie aufgewachsen. Für mich ist das so. Und egal, wer irgendwie Hilfe braucht, bekommt sie. Und das war immer schon so: Wie mein Sohn in den Kindergarten gegangen ist, habe ich mich immer wieder engagiert. Da war es für viele Leute selbstverständlich, dass ich das getan habe. Und jetzt eigentlich, seit die Hilfe für Fremde ist, sag' ich einmal, für Leute, die von weiter her kommen - für Leute, die einen anderen Glauben haben - jetzt plötzlich muss man sich, oder muss ich mich dafür rechtfertigen, dass ich das tue. Mich teilweise auch kritisieren lassen dafür. Habe auch Liebesbriefe an meiner Auslage gefunden. So von wegen: Die gehören alle zurück ins Meer und du gleich dazu. " (Interview mit Freiwilliger/m, Flüchtlingsinitiative Südburgenland, 16.05.2018) 
Auch Auseinandersetzungen mit Hauptamtlichen bezüglich der notwendigen Abgrenzung der Zuständigkeitsbereiche werden in den Interviews erwähnt. So erklärt ein/e Regionalbetreuer/in:

„,Das heißt, was für Wiener Bezieher der Mindestsicherung gilt, gilt nicht für Burgenländer. Und hier wird von den Ehrenamtlichen viel vermischt. Und dann immer: ,Ich hab 'ja gehört ... 'und dieses ,Ich hab ja gehört ... 'wird an die Klienten weitergetragen und die sind dann verunsichert. Und Ehrenamtliche erzählen auch das, was Klienten, wenn sie im Verfahren sind, hören wollen. " (Interview mit Regionalbetreuer/in, 16.05.2018)

Hierin wird deutlich, dass ein Spannungsfeld zwischen haupt- und ehrenamtlich engagierten Personen besteht, da Ehrenamtliche oft im Rahmen persönlicher Gespräche unbeabsichtigt falsche Informationen weitergeben. Gesetze und Erlässe ändern sich in diesem Bereich schnell und es gibt auch viele Unterschiede in der Handhabung der Grundversorgung zwischen den Bundesländern.

HAN-BROICH bestätigt, dass auch in ihrer Studie in Münster Konflikte mit Hauptamtlichen als Grund für die Beendigung der ehrenamtlichen Tätigkeiten angeführt wurden (2012, p. 91 f.). Des Weiteren erwähnt sie, dass Gefühle wie Überforderung, Ärger, Enttäuschung und Frustration auch zum Aufgeben der ehrenamtlichen Tätigkeit führen können.

In den in Oberwart geführten Interviews werden seitens der Ehrenamtlichen neben der Frustration über die fehlenden finanziellen Ressourcen auch Ärger und Enttäuschung erwähnt. Missverständnisse aufgrund kultureller Unterschiede führen laut Interviewpartnerin $\mathrm{C}$ zu negativen Gefühlen, die aber unzureichend artikuliert werden und dann zu einem Verlust der Motivation führen:

„Es gibt so viele - ich hab' das Gefühl, es hat so viele soziale Prozesse gegeben, wo die Einheimischen sich zurückgezogen haben, weil sie nicht verstanden haben, warum das passiert und sich auch die Geflüchteten sich zurückgezogen haben, weil sie nicht verstanden haben, warum das passiert. Und unsere Aufgabe war dann oft noch einmal reden, reden, reden, reden, reden. Warum kommt der nicht zum Training? Weil er nicht verstanden hat in der Gruppe, dass der Wochentag sich geändert hat. [...] Man hat ja dann so eine Distanz, dass man die Konfliktfelder nicht anspricht und sich eher abwendet. Das ist die ,allgemeine Kultur' - also das machen beide. Und dann muss man eben vermitteln und nachfragen, warum war das und schau, das war bei dem so. " (Interview mit Freiwilliger/m Verein SOL, 29.10.2018)

Auch in einem weiteren Beispiel wird die aktive Lösung von Konflikten durch die Involvierung eines/r Koordinator/s/in im Verein als entscheidender Faktor für eine zufriedenstellende ehrenamtliche Tätigkeit hervorgehoben: 
„,Und es ist jetzt auch gut, dass es ihn jetzt gibt als Posten, weil er kann jetzt zwischen Patinnen und Betroffenen vermitteln, weil natürlich nicht alles super toll ist, sondern es gibt natürlich auch Konflikte - das ist ja logisch. Dass er da jetzt den Ausgleich schafft und versucht gemeinsam Lösungen zu finden. Das finde ich schon positiv. " (Interview mit Bildungsberater/in, 08.06.2018)

Im Interview mit einer ehrenamtlich tätigen Person wird noch ein weiterer Grund für die Beendigung der offiziellen Vereinsaktivitäten der Flüchtlingsinitiative Südburgenland erwähnt: Angebote für Geflüchtete würden jetzt nicht mehr benötigt.

„, Aber es ist nicht mehr so - also ich - so wie ich auf meinen eigenen Kind nicht wie eine Glucke sitz, sitz' ich auch bei denen nicht drauf. Weil ich denke, sie müssen sich dann schon - ich hab' ihnen den Weg gezeigt, aber jetzt müssen sie einfach selber weitermachen. " (Interview mit Freiwilliger/m Flüchtlingsinitiative Südburgenland, 16.05.2018)

Im Zitat wird auf den Wandel der Bedürfnisse der Geflüchteten Bezug genommen. In den Augen der Interviewten scheint derzeit ein Zyklus seinen Abschluss zu finden, der 2015 begonnen hat. Damit ist gemeint, dass die Geflüchteten, die 2015/16 in Oberwart angekommen sind, aus Sicht der Freiwilligen ausreichend betreut wurden. Die befragte Person führt im Interview noch weiter aus, sie glaube, dass die wichtigsten Bedürfnisse gedeckt seien und nun die Geflüchteten, die in Oberwart geblieben sind, nur mehr hinsichtlich einiger Kleinigkeiten Hilfe benötigen würden.

Obwohl im Bezirk immer noch Geflüchtete in Grundversorgungsquartieren untergebracht sind, wird im Interview nicht angesprochen, Angebote für nachkommende Geflüchtete zu schaffen oder das Programm spezifisch für kleinere Gruppen umzustrukturieren. Es stellt sich also die Frage, welche Angebote die Asylwerber/innen in Zukunft nützen können, die derzeit im Bezirk Oberwart leben.

Festzuhalten bleibt, dass viele Eigenschaften des neuen Ehrenamts auch in Oberwart sichtbar werden. So tritt beispielsweise dessen projektbezogener Charakter in den Interviews immer wieder indirekt hervor, weil viele der früher Unterstützten keine Betreuung mehr benötigen. Geflüchtete, die später angekommen sind, werden gar nicht mehr angesprochen.

Darin zeigt sich ein Abflauen der Motivation, was jedoch nicht als Indikator für mangelndes Interesse zu werten ist, sondern vielmehr sind es Hindernisse und Hürden, die ein langfristigeres Engagement verhindern.

Um in schwierigen Zeiten die ehrenamtliche Tätigkeit nicht aufzugeben, sind gemäß den Interviews die unterstützende und motivierende Wirkung von Vernetzungstreffen und einer Projektbegleitung wesentlich. Auch HAN-Broich bestätigt diese Überlegung:

„,Eine der wichtigen Unterstützungsleistungen war die Möglichkeit zum Austausch der Ehrenamtlichen untereinander und mit hauptamtlich Tätigen. Hierdurch 
konnten sich eine ,Kommunikationskultur' und ein nachhaltiges , Gruppengefühl' entwickeln, was den Ehrenamtlichen auch half, in schwierigen Zeiten nicht aufzugeben. " (HAN-Broich 2012, p. 43)

Auf diese Art und Weise könnten die eingangs erwähnten Bedürfnisse der Freiwilligen, etwa ,sich weiterzuentwickeln“ und ,interessante Leute zu treffen“ erfüllt werden und die Motivation karitativ tätig zu sein dauerhaft gestärkt werden. Gelingt es, diese Punkte zusammenzuführen, wird eine langfristige Freiwilligenarbeit ermöglicht und gefördert. Beständigere ehrenamtliche Projekte und deren politische Dimension werden im nächsten Abschnitt zu St. Andrä Wördern genauer untersucht.

\section{St. Andrä-Wördern: lokaler Kontext der empirischen Erhebung}

Neben österreichischen rechtlichen Regelungen im Asylbereich und Diskursen zur zunehmenden Betrachtung von Flucht und Migration als Sicherheitsthema (vgl. ScheibelHofer 2017) spielen die spezifischen Rahmenbedingungen einer Gemeinde bei der lokalen Integration von Geflüchteten eine wichtige Rolle. Nach HANNERz kann dabei aber nicht von einer Abgeschlossenheit des Lokalen nach außen ausgegangen werden, sondern eher von einer ,[...] arena in which a variety of influences come together, acted out perhaps in a unique combination, under those special conditions." (HANNERz 1996, p. 27).

Die relevanten äußeren Einflüsse wurden bereits in der Einleitung behandelt, die lokalen Rahmenbedingungen lassen sich für St. Andrä-Wördern folgendermaßen darstellen: Die Marktgemeinde liegt im Bezirk Tulln in Niederösterreich circa 20 Kilometer nordwestlich von Wien. Insgesamt leben in den sieben Katastralgemeinden rund 7.500 Personen. ${ }^{10}$ St. Andrä-Wördern ist durch regelmäßige Zugverbindungen untertags von der Bundeshauptstadt aus mit öffentlichen Verkehrsmitteln gut zu erreichen. Durch die Lage im Umland von Wien ist St. Andrä-Wördern auch nicht von Abwanderung betroffen wie zum Beispiel die Region um Oberwart oder das Mostviertel. Tatsächlich wuchs die Gemeindebevölkerung zwischen den Jahren 2001 und 2018 um rund $22 \% .^{11}$

Bereits seit 1956 gab es im Gemeindegebiet eine Unterkunft für Geflüchtete, in der bis zu 65 Personen untergebracht wurden. Diese wurde von der Österreichischen JungArbeiterBewegung (ÖJAB) geführt und mit 30.06.2018 geschlossen. Nach Renovierungsarbeiten wurde das Quartier im Herbst 2018 als Unterkunft für 48

\footnotetext{
10 Vgl. Gemeindehomepage St. Andrä-Wördern: < https://www.staw.at/Unsere_Gemeinde/ Unser_Ort/Zahlen_Fakten> [Zugriff am 27.12.2018]

${ }_{11}$ Vgl. STAtistiK Austria: < http://www.statistik.at/blickgem/G0201/g32142.pdf> [Zugriff am 18.12.2018]
} 
unbegleitete minderjährige Flüchtlinge (UMF) wiedereröffnet. In der Gemeinde St. Andrä-Wördern existiert außerdem eine recht lange Tradition zivilgesellschaftlichen Engagements in Form des Vereins ,grenzenlos“. Dieser ist vor rund 15 Jahren aus interkulturellen Kochabenden entstanden und versteht sich laut dem derzeitigen Obmann als „lokale Willkommensorganisation“ (Interview mit Obmann ,grenzenlos“, 05.05.2018). Ausgehend von der vorhandenen Diversität im Ort ging es dabei zu Beginn vor allem darum, einen Raum der niederschwelligen Begegnung für Alteingesessene und Zugezogenen aus der näheren und weiteren Umgebung zu schaffen. Geflüchtete Menschen waren dabei von Anfang an zwar ebenfalls gemeint, sie wurden explizit jedoch ab der großen Flüchtlingsbewegung nach Europa 2015 und 2016 involviert.

Wie bereits in der Einleitung beschrieben, entstanden in den Jahren 2015 und 2016 in vielen Städten und Gemeinden Österreichs zivilgesellschaftliche Initiativen zur Unterstützung von Geflüchteten. So auch in St. Andrä-Wördern. Im Herbst 2015 wurden die beiden Initiativen „St. Andrä-Wördern hilft" und „Das Dorf hilft“ von engagierten Personen ins Leben gerufen. In der ersten Phase des Ankommens wurde von den Ehrenamtlichen von „Das Dorf hilft“" vor allem Sachspenden wie Möbel und Kleidung in einem Lager gesammelt und an Geflüchtete verteilt. Zeitgleich versuchte „St. AndräWördern hilft" Geflüchtete bei Behördengängen zu unterstützen und zum Beispiel zu Arztbesuchen zu begleiten. Diese Tätigkeiten entsprechen somit ziemlich genau den Anforderungen der ersten Phase des „Life Cycle of Refugee Integration“ nach SimsA (2017, p. 84).

Einzelne Freiwillige unterstützen Geflüchtete auch individuell in verschiedenen Bereichen wie zum Beispiel bei der Suche nach Wohnraum für anerkannte Asylwerber/innen oder bei rechtlichen Fragen im Asylverfahren mit dem Verweis auf darauf entsprechend qualifizierte Stellen. Vor allem kurz nachdem Geflüchtete in Österreich beziehungsweise in St. Andrä-Wördern angekommen waren, wurde diese Unterstützung von den Ehrenamtlichen als besonders wichtig beschrieben und von den Geflüchteten auch entsprechend angenommen und wertgeschätzt. Vielen Asylwerber/ inne/n und anerkannten Flüchtlingen fehlen in Österreich wichtige soziale Netzwerke, sodass Ehrenamtliche auch als Gatekeeper/innen in die Aufnahmegesellschaft fungieren (LANGTHALER \& Trauner 2009, p. 454 f.). Wie sich in mehreren Gesprächen und Interviews gezeigt hat, nimmt die Bedeutung von ehrenamtlichem Engagement zwar im zeitlichen Verlauf tendenziell ab, bleibt aber doch weiterhin wichtig. Allerdings ändern sich die Bereiche, in denen diese Unterstützung relevant ist. Wenn der erste Bedarf an Sofortmaßnahmen - also zum Beispiel Sachspenden - einmal abgedeckt ist, erhalten die Bereiche Arbeit und Bildung eine größere Wichtigkeit. Aufgrund dieser Verschiebung und der Tatsache, dass in den letzten eineinhalb Jahren signifikant weniger Geflüchtete nach Österreich gekommen sind, besteht auch die Initiative „Das Dorf hilft“ heute in ihrer ursprünglichen Form nicht mehr.

Von Mitgliedern des Vereins „grenzenlos“ gab es bereits vor dem Jahr 2015 Deutschkurse, die von Ehrenamtlichen im ÖJAB-Heim geleitet wurden. Nach einer 
Übergangsphase in der parallel dazu von „St. Andrä-Wördern hilft“ Kurse organisiert wurden, sind diese Bemühungen gebündelt worden. Seit 2017 gibt es einen gemeinsamen Deutschkurs, der dreimal in der Woche in den Räumlichkeiten der Bücherei stattfindet und von Ehrenamtlichen durchgeführt wird. Als gemeindeeigene Integrationsmaßnahme besteht seit 2015 die Möglichkeit für Asylwerber/innen im Rahmen der gesetzlichen Regelungen gemeinnützige Tätigkeiten für den Bauhof zu verrichten. Konkret handelt es sich dabei vor allem um Arbeiten im Bereich der Grünraumpflege. Von dieser Maßnahme profitieren sowohl Geflüchtete, da sie eine sinnvolle Beschäftigung und zusätzliche finanzielle Mittel erhalten, aber auch die Gemeinde, die dadurch weitere Projekte in diesem Bereich umsetzen kann. Abgesehen von der Möglichkeit, gemeinnützige Tätigkeiten bei der Gemeinde zu verrichten, ist es Asylwerber/inne/n mit wenigen Ausnahmen nicht möglich am österreichischen Arbeitsmarkt teilzuhaben. Allerdings sind einige bereit, sich selbst ehrenamtlich zu engagieren, zum Beispiel beim Roten Kreuz oder der Flurreinigungsaktion in der Gemeinde. Meistens kommen diese Kontakte auch mit Unterstützung von Ehrenamtlichen zustande. Was den Bildungsbereich betrifft, ist vor allem der Deutschkurs in St. Andrä-Wördern von zentraler Bedeutung. Dabei geht es jedoch nicht nur um Sprachvermittlung, sondern auch um Unterstützung in einem viel weiteren Sinn, wie eine/r der Deutschlehrer/innen berichtet:

„[...] der Deutschkurs ist natürlich eine Bildungsmaßnahme, aber der ist durch und durch integrativ. Es ist einfach ein soziales Netz, wo du mit allen Problemen und Sorgen hinkommen kannst und wo du alle Informationen bekommst und Hilfe und Unterstützung. “ (Interview ehrenamtliche Deutschlehrerin, 23.05.2018)

\subsection{Arbeitsteilung auf lokaler Ebene in St. Andrä-Wördern}

Bei der Integration von Geflüchteten in St. Andrä-Wördern stellten sich die Personen, die sich ehrenamtlich engagieren, als entscheidender Faktor heraus. Interessant in diesem Zusammenhang ist die Art und Weise der Zusammenarbeit zwischen den unterschiedlichen Akteur/inn/en. Hier kann beobachtet werden, dass ehrenamtlich engagierte Personen in gewisser Weise eine Lücke füllen, die dadurch entsteht, dass staatliche Stellen nicht fähig oder willens sind, ihre Verantwortung im Bereich der Versorgung und Integration von Geflüchteten angemessen zu erfüllen (PrIEs 2018; Cantat \& Feischmidt 2018, p. 396). Auffallend ist außerdem, dass diese Aufgaben im Fall von St. Andrä-Wördern nicht von den großen etablierten NGOs in diesem Feld, sondern eben von zivilgesellschaftlichen Initiativen und Vereinen übernommen wurden. Dies zeigt sich auch in der Aufgabenteilung auf lokaler Ebene. Während die Zivilgesellschaft vorwiegend den operativen Teil der Umsetzung von Integrationsmaßnahmen übernimmt, versucht die Gemeinde diese Bemühungen mit den zur Verfügung stehenden Mitteln zu unterstützen. So können die Ehrenamtlichen zum Beispiel die Räumlichkeiten der Bücherei mietfrei für die Deutschkurse nutzen oder auf dem Gemeindeamt 
kostenlos Unterlagen kopieren. Außerdem wurde über die Gründung der Initiativen und deren Aktivitäten auch immer wieder im Amtsblatt von St. Andrä-Wördern berichtet. Es gab somit auch eine entsprechende Öffentlichkeitsarbeit seitens der Gemeinde in diesem Bereich.

Allerdings würden sich einige der Ehrenamtlichen mehr Unterstützung von Gemeindeseite wünschen. In Interviews wurde immer wieder betont, dass die Integration von Geflüchteten in St. Andrä-Wördern im Allgemeinen recht gut funktioniert. Dazu notwendig ist auf der einen Seite das beharrliche Engagement der Zivilgesellschaft und auf der anderen Seite die Bereitschaft der Gemeinde dieses zu befürworten. Auch wenn ein Großteil der Aktivitäten zur Integration von Geflüchteten darauf beruht, dass Menschen ehrenamtlich Zeit investieren und Geflüchtete auf vielfältige Art und Weise unterstützen, ist dazu auch ein gesellschaftliches Klima notwendig, welches dieses Engagement unterstützt.

Während somit ein Großteil der Unterstützung für Geflüchtete von Freiwilligen bereitgestellt wird, sieht es die Gemeinde durchaus als ihre Aufgabe an, sich mit dem kommunalen Diskurs auseinanderzusetzen und bei Bedarf auch entsprechend aktiv zu werden. Als sich gegen die Unterbringung von UMF in einem weiteren Quartier in St. Andrä-Wördern Widerstand regte und eine Unterschriftenliste gegen dieses Projekt gestartet wurde, war auch die Gemeinde gefordert damit umzugehen. Hier zeigt sich, dass zivilgesellschaftliches Engagement im Bereich Flucht und Migration nicht nur von Gruppen ausgeübt wird, die bereit waren Geflüchtete zu unterstützen (PRIES 2018, p. 5 f.; YoukHana \& SUTter 2017) Gemeindevertreter/innen versuchten in persönlichen Gesprächen mit den Initiator/inn/en und Unterstützer/inne/n der Unterschriftenaktion auf deren Bedenken zu reagieren. Außerdem wurde eine Diskussionsveranstaltung mit Vertreter/inne/n der Zivilgesellschaft, Unterstützer/inne/n von Geflüchteten und Lokalpolitiker/inne/n organisiert. Nach einigen Problemen mit den UMF in der Unterkunft sind in diesem jetzt eine Familie und mehrere erwachsene Männer untergebracht, die Aufregung hat sich inzwischen gelegt. Die Verantwortlichkeit der Gemeindevertreter/ innen für lokale Integration ist hier eher eine gesamtgesellschaftliche und bezieht sich weniger auf Geflüchtete an sich. Dabei gilt es auch einen Mittelweg zu finden zwischen den Forderungen der einen Seite und den Bedenken der anderen Seite. Die unterschiedlichen Zugangsweisen und Aufgabengebiete der verschiedenen Akteur/inn/e/n bilden somit den Rahmen, innerhalb dessen sich die politischen Dimensionen des Engagements von Ehrenamtlichen in der Gemeinde entfalten.

\subsection{Politische Dimensionen des Engagements von Freiwilligen}

Zivilgesellschaftliche Aktivitäten im Feld von Flucht und Integration können grob in zwei unterschiedliche Zugänge unterteilt werden (DE JonG \& ATAç 2017:, p. 30). Im „problem-solving approach“ geht es vor allem darum Hilfestellungen für Geflüchtete, wie zum Beispiel Sachspenden, Beratungen oder Unterkünfte, bereitzustellen während 
der „critical approach“ vor allem von Gruppierungen oder Einzelpersonen vertreten wird, die sich mit Geflüchteten solidarisch erklären, sich für deren Rechte einsetzen oder versuchen politischen Druck auf Regierungen auszuüben. Die meisten ehrenamtlichen Tätigkeiten in St. Andrä-Wördern können somit dem ,problem-solving approach“ zugeordnet werden. Obwohl darin selten eine explizit politische Intention mitschwingt (vgl. Fleischmann \& Steinhilper 2017, p. 19 f.), gehen damit doch Auswirkungen einher, die alles andere als unpolitisch sind. Es zeigt sich also, dass diese klare Unterscheidung zwischen humanitärer Hilfe zur Unterstützung von Menschen und politischem Aktivismus als Engagement gegen repressive Strukturen für die Akteur/inn/e/n in St. Andrä-Wördern nicht zielführend ist (vgl. CANTAT \& FEISCHMIDT 2018, pp. 390 f.). Vielmehr gilt es, hier ein differenzierteres Bild zu entwerfen.

Viele der Ehrenamtlichen entwickeln infolge ihres unmittelbaren Kontakts zu Geflüchteten und durch die Erfahrungen, die sie bei der Unterstützung derselben im Lauf der Zeit gemacht haben, eine kritische Haltung gegenüber dem österreichischen Asylregime und den restriktiven Rahmenbedingungen, mit denen Asylwerber/innen in Österreich zu kämpfen haben (vgl. Fleischmann \& Steinhilper 2017, p. 22; HamanN \& KARAKAYAli 2016, p. 80). Die Unterstützung von Geflüchteten ist daher auch eine gewisse Form von Widerstand gegen diese Rahmenbedingungen und diskriminierenden Diskurse in Österreich. Aus dieser Perspektive kann das Schaffen von Räumen für Begegnungen zwischen Geflüchteten, Migrant/inn/en und Österreicher/inne/n, wie zum Beispiel bei den Kochabenden oder den Deutschkursen, als gesellschaftskritische Praxis mit einer durchaus politischen Dimension verstanden werden (vgl. De Jong \& Атас̧ 2017, p. 34 f.). Einerseits wird auf dem Recht der Zuerkennung von öffentlichen Räumen für Menschen bestanden, in welchen Staatsbürgerschaft und Herkunft keine Rolle spielen. Andererseits wird damit auch der „,organisierten Desintegration“ (TÄUBIG 2009), Isolation und Segregation vor allem von Asylwerber/inne/n entgegengewirkt. (De Jong \& Aтас̧ 2017, p. 34 f.). Die zivilgesellschaftlichen Aktivitäten in St. AndräWördern können somit auch als „subversive humanitarianism“ nach VANDEVOORT \& Verschraegen (2018) beschrieben werden, die darunter Folgendes verstehen:

,... a morally motivated set of actions, which acquires a political character not through the form in which these actions manifest themselves, but through their implicit opposition to the ruling socio-political climate. " (VANDEVOORT \& VersCHRAEGen 2018, p. 105)

Ausgehend von einer Beschreibung der lokalen Rahmenbedingungen, den Akteur/ inn/en und deren Aktivitäten bei der Integration von Geflüchteten in St. Andrä-Wördern zeigte sich eine spezifische Form der Aufgabenverteilung auf kommunaler Ebene. Die Initiativen „Das Dorf hilft“ und „St. Andrä-Wördern hilft“ in Zusammenarbeit mit dem Verein „grenzenlos“ stellen dabei relativ junge Formen zivilgesellschaftlichen 
Engagements dar ${ }^{12}$ deren Aktivitäten als „subversive humanitarianism“ entscheidend zum Gelingen von Integration auf lokaler Ebene beitragen, aber auch in den damit verbundenen Diskursen in der Gemeinde eine wichtige Rolle spielen. Gleichzeitig wird in diesen Tätigkeiten aber auch auf nationale Rahmenbedingungen für Geflüchtete in Österreich Bezug genommen und werden deren Auswirkungen auf die Lebensrealität von Asylwerber/inne/n und Asylberechtigten in der Gemeinde sichtbar gemacht. Folgendes Zitat fasst diese Zugangsweise recht prägnant zusammen:

„,Also mir geht's um ein gutes Zusammenleben, [...] das ist meine Motivation. Mit Geflüchteten, aber auch überhaupt diese Vielfalt, die es im Ort gibt, als Ressource zu sehen. [...] [Dass es] da ein gutes Zusammenleben gibt und eine Willkommenskultur und nicht so eine grade so aktuelle Ablehnungskultur, wie wir sie von der Politik vorgelebt kriegen. “ (Interview mit dem Obmann des Vereins ,grenzenlos“, 05.05.2018)

Im Vorangegangenen wurde unter anderem auf die Möglichkeiten der Unterstützung von Ehrenamtlichen durch die Gemeinde eingegangen. Auch auf der Ebene der Bundesländer kann sich aktive Integrationspolitik positiv auf lokale Initiativen in Gemeinden auswirken. Vor allem Oberösterreich ist in diesem Zusammenhang hervorzuheben, da dort ein weitreichendes Netz mit eigens eingerichteten regionalen Zentren für Integration aufgebaut wurde.

\section{Politische Strukturen zur Förderung der Integration in Oberösterreich}

Die stark ansteigende Zahl von Asylanträgen ab 2015 stellte die staatlichen Strukturen vor große Herausforderungen. Logistische und finanzielle Engpässe behinderten die Arbeit. Es mangelte an Grundversorgungsquartieren und ausreichendem Personal für die Betreuung der Geflüchteten. Ehrenamtliche Helfer/innen fingen einen großen Anteil der anfallenden Aufgaben ab (vgl. DAPHI 2016). Ohne das Engagement der freiwilligen Helfer/innen wäre es nicht möglich gewesen, die Anforderungen der Flüchtlingsaufnahmen auf dem letztendlich erreichten Niveau zu bewältigen (AUMÜLLER 2016, pp. 2 f., 6). Große Herausforderungen für das zivilgesellschaftliche Engagement waren vor allem Informationsdefizite und sich laufend ändernde Rahmenbedingungen. Eine funktionierende Kooperation zwischen Zivilgesellschaft, NGOs und Behörden ist für eine erfolgreiche Integration notwendig. Die österreichische Integrationspolitik ist nicht nur Bundessache, viele Entscheidungen hinsichtlich einer Strukturierung von Integration werden in den einzelnen Ländern getroffen. Dadurch entsteht zwischen den

12 Wie bereits an anderer Stelle erwähnt, gibt es den Verein „grenzenlos“ schon viel länger, aber viele Personen aus dessen Umfeld engagierten sich auch in den neu gegründeten Initiativen, sodass es weder personell noch inhaltlich starke Trennlinien in diesem Zusammenhang gibt. 
Bundesländern eine große Inhomogenität, was die Strukturen der Integration und die Vernetzung der einzelnen Akteur/inn/e/n und Organisationen betrifft.

In diesem Abschnitt liegt der Fokus auf den spezifischen Integrationsstrukturen von Oberösterreich. Diese heben sich stark von denen anderer österreichischer Bundesländer $a b$ und können als Best-Practice-Modell gesehen werden, in dem ehrenamtliche und öffentliche Institutionen durch intensive Vernetzung erfolgreich zusammenarbeiten. Schon 2008 wurde in Oberösterreich ein detailliertes Integrationsleitbild mit dem Titel „Einbeziehen statt Einordnen. Zusammenleben in Oberösterreich“ verfasst (LAND OÖ 2008, p. 23). Es wurde die damalige Situation in Oberösterreich untersucht und daraufhin ein strategischer Maßnahmenplan unter Einbindung aller gesellschaftlichen Akteur/inn/e/n und Multiplikator/inn/en entwickelt (LAND OÖ 2008, p. 5).

\begin{abstract}
„,Oberösterreich weist im Vergleich zu anderen Bundesländern eine hohe Zahl an speziellen integrationsfördernden Stellen in den einzelnen Regionen auf. Diese Einrichtungen (v.a. Integrationsbüros, Integrationszentren und regelmäßige Sprechtage von Caritas, Volkshilfe und dem Verein migrare) werden vom Land OÖ finanziell gefördert und stellen einen großen Vorteil für aktuelle und zukünftige regionale Integrationsmaßnahmen dar. "(LAND OÖ 2008, p. 40)
\end{abstract}

\title{
5.1 Vernetzung zur Informationsweitergabe
}

Mit der Umsetzung der im Integrationsleitbild entworfenen Maßnahmen wurde allerdings erst im Herbst 2015 begonnen, nachdem die neue oberösterreichische Landesregierung die Zuständigkeit für die Themen Asyl und Integration an Landesrat Rudi Anschober übertrug (Amt DER OÖ. LANDESREGIERUNG 2016, p. 1). Der Fokus lag zunächst auf der Schaffung neuer Quartiersplätze. Zu diesem Zweck wurde Mitte November 2015 die Initiative „Zusammen Helfen in Oberösterreich. Hilfe für Menschen auf der Flucht“ gegründet. Neben NGOs wie Volkshilfe, Caritas und Diakoniewerk unterstützten auch das Rote Kreuz, der Samariterbund, die Arbeiterkammer OÖ, die Wirtschaftskammer OÖ und die Landwirtschaftskammer OÖ die Initiative (AMT DER OÖ. LANDESREGIERUNG 2015, pp. 1 f., 4 ff.).

Im oberösterreichischen Integrationsleitbild von 2008 wird die Einrichtung von Integrationsausschüssen auf Landes- und Bezirks- bzw. Gemeindeebene empfohlen. Es wurde im Integrationsleitbild festgestellt, dass der Austausch zwischen Vertreter/inne/n unterschiedlichster Institutionen, der gegenseitige Wissenstransfer und das Kennenlernen von Ansprechpersonen für jeweils relevante Themenbereiche eine wichtige Rolle zur Förderung erfolgreicher Integration einnimmt (LAND OÖ 2008, p. 39). Zu diesem Zweck wurde 2016 von Landesrat Anschober die Steuerungsgruppe des Landes eingerichtet. Diese findet seither zweimal wöchentlich statt und dient als Informa- 
tionsdrehscheibe. Maßnahmen werden beschlossen und die landesweite Umsetzung von Integration wird koordiniert (OÖ INTEGRATIONSRESSORT 2017, p. 3). Neben Vertreter/ inne/n des Landes Oberösterreich (Integrationsstelle OÖ, Grundversorgung, Wirtschaft und Bildung) sind auch weitere wichtige Stakeholder, wie die Bereichsleitungen der Regionalen Kompetenzzentren für Integration und Diversität (ReKIs), der Sprecher der Bezirkshauptleute, die politischen Büros der Landesregierung bzw. der Stadt Linz, die in der Flüchtlingsbetreuung tätigen NGOs, das AMS, der Landesschulrat, das Bundesamt für Fremdenwesen und Asyl (BFA), die Plattform Zusammen Helfen in Oberösterreich sowie die Exekutive aktiv (InTEGrationsstelle \& Grundversorgungsstelle OÖ 2017, p. 18).

„In Oberösterreich haben wir einfach ein sehr breites Netzwerk. [...] Und das ist das eigentliche Erfolgsgeheimnis glaub'ich, dass da alle gemeinsam in einem Team arbeiten. Das wird abgestimmt aufeinander. Da ist eine Respekts- und Vertrauensebene da, da ist so ein richtiger, ein Teamcharakter da. So unter dem Motto, wir haben ein gemeinsames Ziel und jeder versucht in seinem Beitrag bestmöglich daran zu arbeiten, dass das verwirklicht wird." (Interview mit Landesrat Anschober, 16.10.2018)

Alle Informationen aus der Landessteuerungsgruppe werden an die Bezirkssteuerungsgruppen weitergegeben. Die Bezirkssteuerungsgruppen wurden 2015 vorrangig gegründet, um bei der landesweiten Schaffung neuer Grundversorgungsquartiere zu unterstützen. Inzwischen geht aber die Zahl der Asylanträge stark zurück und Grundversorgungsquartiere werden teilweise wieder geschlossen, weshalb diese Steuerungsgruppen eine Koordinierungsfunktion bei der Umsetzung der Integrationsarbeit in den einzelnen Bezirken übernehmen (OÖ INTEGRATIONSRESSORT 2017, p. 3). Der Vertreter aller oberösterreichischer Bezirkshauptmannschaften in der Landessteuerungsgruppe meinte dazu in einem Interview:

„,Und so werden Dinge einerseits von Bund und Land in die Bezirke reingespielt oder umgekehrt, von unserer Seite [...] auch Richtung Land oder Bund transportiert. “ (Interview mit dem Bezirkshauptmann eines Bezirkes, 26.07.2017)

Auf Gemeindeebene, abhängig davon ob es ein Asylquartier gibt oder nicht, werden ebenfalls Steuerungsgruppen zur Umsetzung der Integrationsmaßnahmen in den Gemeinden gebildet (OÖ INTEGRATIONSRESSORT 2017, p. 3).

„Und das [...] ist eben die kommunale Ebene, das ist eben das, wo Oberösterreich auch einen Schwerpunkt darauf legt, dass Integration auf der Gemeindeebene stattfindet. Also, da ist das Zusammenleben, und da gibt es natürlich ganz spannende Möglichkeiten, sozusagen dieses Miteinander zu stärken. “ (Interview mit eine/r Mitarbeiter/in der Volkshilfe OÖ, ReKI, 10.09.2018) 


\subsection{Strukturierung des freiwilligen Engagements vor Ort - ReKIs}

Seit Jänner 2015 wurden in allen Bezirken Oberösterreichs „Regionale Kompetenzzentren für Integration und Diversität" (ReKIs) eingerichtet. Das Projekt wurde von der Integrationsstelle des Landes Oberösterreich beauftragt und ist auf zwei Trägerorganisationen aufgeteilt. Die Volkshilfe ist zuständig für sieben Bezirke und die Caritas ist als Partnerorganisation für die restlichen acht Bezirke in Oberösterreich verantwortlich (Interview mit eine/r Mitarbeiter/in der Volkshilfe OÖ, ReKI, 10.09.2018). Der primäre Aufgabenbereich ist die regionale und kommunale Prozessbegleitung.

Das Grundmandat der ReKIs besteht in der strategischen Begleitung von Gemeinden bei deren Integrationsarbeit. Zunächst waren sie vor Ort dafür zuständig die Gründung von Flüchtlingsquartieren zu ermöglichen. Es wurden verschiedene Informationsveranstaltungen für die lokale Bevölkerung organisiert, um diese miteinzubeziehen und so das gesellschaftliche Fundament für Integration von Anfang an zu legen. Gemeindesteuerungsgruppen wurden eingerichtet (Interview mit eine/r Mitarbeiter/in der Volkshilfe OÖ, ReKI, 10.09.2018). Zusätzlich zu den Informationsveranstaltungen wurden Workshops für jene angeboten, die sich zur Unterstützung der Geflüchteten in den Gemeinden bereit erklärt haben. Dabei wurde die Zivilgesellschaft bei der Bildung eigener Strukturen vor Ort durch die ReKIs und die Integrationsstelle OÖ unterstützt. So wurde erreicht, dass die jeweiligen Akteur/inn/e/n gut strukturiert je nach dem unmittelbaren Bedarf tätig sein können. Zusätzlich wurde in den Gemeinden eine Steuerungsgruppe zur Koordinierung der Integration der Asylwerber/innen installiert. Diese Steuerungsgruppen bestehen aus Ansprechpersonen der jeweiligen Arbeitsgruppen, aus Vertreter/inne/n der Gemeinden, Quartierbetreiber/inne/n und in manchen Fällen auch aus weiteren Schlüsselpersonen aus der Zivilgesellschaft, wie etwa Pfarrern oder Vereinsobleuten (InTEgrationsstelle \& Grundversorgungsstelle OÖ 2017, p. 19 f.).

„Großteils sind das noch Strukturen die 2015/2016 entstanden sind. [Es] wurden Gemeindesteuerungsgruppen geschaffen, um freiwilliges Engagement und Quartiergeber an die Gemeinde als Institution zu binden. Um kurze Ent scheidungswege zu haben, um Abstimmungen zu ermöglichen und um den Informationsfluss zu garantieren. Das ist das, wo im Bereich Asyl sehr viel passiert ist. Wo viel Initiative entstanden ist, und wo es auch relativ leicht war, da Freizeit-, Sport-, und Musikvereine irgendwie einzubinden und auf der Gemeindeebene eben für Partizipation und Teilhabe zu sorgen. "(Interview mit eine/r Mitarbeiter/in der Volkshilfe OÖ, ReKI, 10.09.2018) 


\section{3 „Zusammen Helfen in Ö̈“-Die Rolle der Zivilgesellschaft bei der Integration}

Ehrenamtliche erfüllen wichtige Aufgaben bei der Integration von Geflüchteten, insbesondere jene, die langfristig engagiert sind. In der zukünftigen Planung von Integrationspolitik und Integrationsmaßnahmen sollten Ehrenamtliche miteinbezogen und konsultiert werden. Eine engagierte Zivilgesellschaft kann positiv zur Integration beitragen, benötigt dafür aber politischen Rückhalt. Die vielen Projekte und Initiativen, die angesichts der Erfordernisse von 2015 und 2016 entstanden sind, müssen von staatlicher Seite und von Stiftungen gefördert werden, um nachhaltig zivilgesellschaftliche Strukturen etablieren zu können, die offen und integrativ wirken (KARAKAYALI \& KLEIST 2015, p. 5 f.). Eine Kooperation zwischen Zivilgesellschaft und staatlichen Stellen ist wichtig, um gute Integrationsarbeit leisten zu können. Das oberösterreichische Modell der Vernetzung ist ein Best-Practice Beispiel dafür, wie die Kommunikation und der Austausch zwischen Zivilgesellschaft, NGOs und Behörden funktionieren können.

„Über 10.000 HelferInnen sind in OÖ mittlerweile für die Integration aktiv und stellen vielfach die BrückenbauerInnen in den Gemeinden dar. Als Anlaufstelle für die Engagierten dienen ZusammenHelfen in $O \ddot{O}$ und die Integrationsstelle OÖ. Die HelferInnen sind für die Integration von entscheidender Bedeutung. " (OÖ INTEGRATIONSRESSORT 2017, p. 5)

Im Herbst 2015 übernahm die Hilfe für Geflüchtete vor allem Erstversorgung und Akuthilfe. Die Tätigkeitsfelder wurden aber mit der Zeit ausgedehnt auf Sachspenden, Übersetzungsarbeit, Wohnraum, Lernen/Bildung, Rechtsberatung, Freizeitgestaltung, Unterstützung bei Behördenwegen, Gesundheit und Arbeitsmarkt. All diese Themenbereiche werden inzwischen durch zivilgesellschaftliches Engagement abgedeckt (SIMSA et al. 2016, p. 16). Um die Vielzahl an freiwilligen Aktivitäten von ehrenamtlich engagierten Gruppen und deren Angebote vernetzen zu können und die Informationen einer breiten Öffentlichkeit zugänglich zu machen, veröffentlichte die Initiative von Landesrat Anschober „Zusammen Helfen in OÖ“ auf ihrer Homepage ${ }^{13}$ eine Landkarte in der oberösterreichweit bereits über 120 Freiwilligenorganisationen enthalten waren. Mit Stand Jänner 2017 waren in Oberösterreich 262 freiwillige Initiativen rund um das Thema Asyl und Integration aktiv (InTEGrationsstelle \& GRUNDVERSORGungsstelle OÖ 2017, p. 19).

Die Zivilgesellschaft übernimmt die Aufgabe der Schaffung eines engmaschigen Netzes von direkten Kontakten zwischen den Geflüchteten und der lokalen Bevölkerung (Simsa et al. 2016, p. 3). Durch die starke Präsenz des Landes entstehen Strukturen, die einen niederschwelligen Zugang zu Informationen ermöglichen und Vernetzung erleichtern.

13 Vgl. $<$ https://zusammen-helfen.at/freiwillige-initiativen-in-oberoesterreich/> [Zugriff am 09.01.2019]. 


\begin{abstract}
„,Wir haben wenig direkt mit Geflüchteten zu tun. Wo wir schon eine Rolle spielen, das ist einfach in diesem schon sehr starken zivilgesellschaftlichen Engagement, das eben gerade 2015/ 2016 entstanden ist. Und wir haben eine große Rolle dabei gehabt dieses zivilgesellschaftliche Engagement zu unterstützen. Also arbeitsfähige Strukturen zu schaffen, die Kommunikation zur Gemeinde sicherzustellen, die Informationen von Landes- und Bezirksebene auf die Gemeindeebene zu den freiwillig Engagierten auch zu bringen. Und wir haben auch über ein Expert/inn/ en-Pool-Angebot der Integrationsstelle Oberösterreich, sozusagen Schulungen für diese Ebene angeboten für die freiwillig Engagierten. " (Interview mit eine/r Mitarbeiter/in der Volkshilfe OÖ, ReKI, 20.09.2018)
\end{abstract}

Die Zivilgesellschaft hat im Rahmen der Bewältigung der Situation 2015/16 einen wichtigen Beitrag geleistet. Durch die Veränderung der Hilfeleistungen, die zunächst vor allem in Form von Erstversorgung und Akuthilfe erbracht wurden, ist es notwendig geworden, das zivilgesellschaftliche Engagement zu unterstützen, um dieses Potenzial ausschöpfen zu können. Da inzwischen auch sehr spezifische Hilfeleistungen, wie bei der Wohnungs- und Arbeitssuche und Unterstützung bei rechtlichen Fragen, durch die Aufnahmegesellschaft übernommen werden, ist es notwendig diese Informationen durch Veranstaltungen bereitzustellen. Oberösterreich bietet diesbezüglich durch Konferenzen zu verschiedenen Themen, kostenlose Rechtsberatung, Workshops und Hilfestellungen vor Ort in den Gemeinden ein vielfältiges Unterstützungsangebot (INTEGRATIONSSTELLE \& Grundversorgungsstelle OÖ 2017, p. 22). Durch die vorhandenen Strukturen funktionieren Informationsweitergabe und Austausch mit ehrenamtlichen Initiativen. Das enge Netzwerk in Oberösterreich erleichtert die Zusammenarbeit und bietet detaillierte Informationen darüber, wo welche Ansprechpartner/innen für welche Fragen zu finden sind. Dadurch können die einzelnen Akteur/inn/e/n, die für die Integration von Geflüchteten aktiv sind, kraftvoller an einem Strang ziehen.

\title{
6 Dimensionen ehrenamtlichen Engagements für Geflüchtete
}

Die Abschnitte über das Mostviertel, die Region um Oberwart und die Gemeinde St. Andrä-Wördern beschäftigen sich mit den umfangreichen Tätigkeiten von Ehrenamtlichen im Zusammenhang mit der Integration von Geflüchteten. Für Oberösterreich werden die relevanten Strukturen in diesem Bereich beschrieben. Abschließend sollen vier unterschiedliche Dimensionen zivilgesellschaftlichen Engagements für Geflüchtete herausgearbeitet werden, die aus den Beispielen hervorgehen. Erstens hat dieses Engagement eine emotionale Dimension, wie sie vor allem für die Gemeinden des Mostviertels beschrieben wurde. Geflüchtete und Unterstützer/innen gehen vielfältige Beziehungsformen zueinander ein. Durch Kontakte und Austausch werden 
beidseitige Lernprozesse in Gang gesetzt. Der Aufbau persönlicher Beziehungen stärkt die Wahrnehmung der Geflüchteten in der Gemeinde willkommen zu sein. Außerdem stellen die so aufgebauten Kontakte eine wichtige Möglichkeit für den Einstieg in die österreichische Gesellschaft dar. Des Weiteren hat zivilgesellschaftliches Engagement eine politische Dimension, wie im Beitrag zu St. Andrä-Wördern erläutert wird. Das Schaffen von Räumen für niederschwellige Kontakte zwischen Geflüchteten und der Gemeindebevölkerung ist imstande die Isolation und Ausgrenzung vor allem von Asylwerber/inne/n zumindest zu einem gewissen Grad zu überwinden. Die dabei entstehenden Netzwerke sind auch oft eine entscheidende Ressource für den Arbeitsmarktzugang und die Bildungsmöglichkeiten für Geflüchtete. Das Engagement für Geflüchtete in Österreich seit 2015/16 weist als innovative Spielart des „,neuen Ehrenamtes“ auch eine interessante motivationale Dimension auf. In Oberwart, aber auch in vielen anderen Regionen Österreichs sind engagierte Personen abseits der bereits etablierten NGOs in Eigeninitiative aktiv geworden und haben selbstständig Gruppierungen zur Unterstützung von Geflüchteten gebildet. Im Kontext dieser Prozesse haben sich im Lauf der Zeit auch immer wieder Hindernisse aufgetan, welche unter anderem durch eine externe Projektbegleitung gelöst werden konnten. In einer strukturellen Dimension versucht das Land Oberösterreich genau diese Unterstützung für Ehrenamtliche bereitzustellen. Durch ein dichtes Netz an lokalen Koordinationsstellen werden notwendige Informationen für das kontinuierliche Engagement leicht zugänglich bereitgestellt. Es sind klar definierte Auskunftspersonen vorhanden, die in unterschiedlichen Bereichen unterstützen können. Auch die Vernetzung der einzelnen Initiativen wird auf diese Weise gefördert.

Ausgehend von der Initiative engagierter Personen in den Jahren 2015/16 bildeten sich mit der Zeit Kooperationen, ein reger Informationsaustausch und unterstützende Strukturen. Rahmenbedingungen, um dieses Engagement zu fördern, wie zum Beispiel in Oberösterreich sind erst später hinzugekommen. Auch sind diese Strukturen in der Form österreichweit einzigartig. Hierin wird deutlich, dass der politische Wille zur Gestaltung von Integrationsprozessen ebenfalls eine wichtige Rolle spielt. Auch in St. Andrä-Wördern hat sich gezeigt, dass ehrenamtliche Initiativen von infrastruktureller Unterstützung profitieren und sich ein Bewusstsein für die Integrationsthematik in der Gemeinde sehr positiv auswirkt.

\section{Conclusio und Ausblick}

Im Lauf der Zeit ändern sich nicht nur, wie bereits beschrieben, die Rahmenbedingungen von ehrenamtlichem Engagement für Geflüchtete, sondern auch die damit verbundenen Herausforderungen. Mit der Verschiebung von einer anfänglichen Willkommenskultur hin zu einer tendenziell eher negativen Stimmung Geflüchteten gegenüber müssen sich Ehrenamtliche auch immer wieder für ihre Tätigkeiten rechtfertigen, wie das Beispiel aus Oberwart zeigt. Dadurch, dass die Zahlen der Geflüchteten, die in den 
Gemeinden untergebracht sind rückläufig sind, werden auch viele während der Jahre 2015/16 gegründete Initiativen nicht mehr in ihrer ursprünglichen Form weitergeführt. Die Gründe hierfür sind vielfältig. Ein entscheidender Faktor in vielen Fällen ist jedoch die Tatsache, dass Geflüchtete nach dem Abschluss des Verfahrens vor allem aus dem ländlichen Raum häufig in größere Städte, insbesondere nach Wien, ziehen. In diesem Zusammenhang spielen sowohl das Angebot an Arbeitsplätzen, die Möglichkeiten zur Mobilität, höhere Sozialleistungen in Wien, aber auch individuelle Präferenzen von Geflüchteten im urbanen Raum wohnen zu wollen sowie die dort vorhandenen ,ethnic communities" eine Rolle.

Es stellt sich abschließend die Frage, in welche Richtung sich ehrenamtliches Engagement für Geflüchtete in Österreich entwickeln wird. In Oberwart und den Gemeinden im Mostviertel wurden einige Initiativen inzwischen wieder aufgelöst, es gibt aber noch einzelne engagierte Personen, die jene Geflüchteten unterstützen, die noch in der Region leben. Im Gegensatz dazu existieren in St. Andrä-Wördern nach wie vor ehrenamtliche Vereine zur Unterstützung von Geflüchteten. Die Kochabende von „grenzenlos“ bilden dabei auch einen sozialen Raum für gemütliches Beisammensein unter Gleichgesinnten, in dem Begegnungen auf Augenhöhe stattfinden können und wo Geflüchtete nicht auf ihre Herkunft oder ihren rechtlichen Status in Österreich reduziert werden.

Durch den persönlichen Kontakt mit Geflüchteten haben Ehrenamtliche in den vergangenen drei Jahren einen unmittelbaren Einblick in die Lebenswelt von Asylwerber/ inne/n und Asylberechtigten erhalten und Freundschaften und Beziehungen aufgebaut. Diesen Erfahrungen stehen Gefühle von Machtlosigkeit und Enttäuschung gegenüber, wenn Asylwerber/innen lange auf den Ausgang des Verfahrens warten müssen oder trotz gelungener Integration in der Gemeinde abgeschoben werden. Was bleibt, ist der entscheidende Beitrag, der von Ehrenamtlichen geleistet wurde, um das Ankommen für Geflüchtete in Österreich zu erleichtern.

\section{Quellenverzeichnis}

\subsection{Literaturverzeichnis}

AMt DER OÖ. LANDESREgIERUNG (2015), Herbergssuche 2015/ 2016: Start der Initiative ZUSAMMEN.HELFEN IN OÖ für mehr Asylquartiersplätze mit Unterstützung aus ganz Oberösterreich. URL: <https://www.land-oberoesterreich.gv.at/Mediendateien/LK/ PKAnschober20112015InternetNEU.pdf> [Zugriff am 07.01.2019]

Amt der OÖ. LANDESREgIERUNG (2016), Gemeinsam schaffen wir das! Das ungeliebte, aber vielleicht aktuell wichtigste Ressort der Landesregierung: Erste Zwischenbilanz des neuen Integrationsressorts nach einem Jahr Arbeit. Information zur Pressekonferenz mit Landesrat Rudi Anschober. 
URL: $\quad<$ http://www.anschober.at/index.php?id=39\&tx_ttnews[tt_news] $=3288>$ [Zugriff am 07.01.2019]

AuMÜLLER J. (2016), Flüchtlingszuwanderung und bürgerschaftliches Engagement. In: betrifft: Bürgergesellschaft 42. Friedrich-Ebert-Stiftung.

URL: <https://www.fes.de/buergergesellschaft/publikationen/documents/BB-42Fluechtlin gszuwanderungInternet.pdf $>$ [Zugriff am 07.01.2019]

Cantat C. \& Feischmidt M. (2018), Conclusion: Civil Involvement in Refugee Protection Reconfiguring Humanitarianism and Solidarity in Europe. In: Feischmidt M., Pries L. \& Cantat C. (eds.), Refugee Protection and Civil Society in Europe. Cham, Palgrave Macmillan, pp. 379-399.

DAPHI P. (2016), Zivilgesellschaftliches Engagement für Flüchtlinge und lokale ,,Willkommenskultur". Bundeszentrale für politische Bildung. 1.4.2016.

URL: <http://www.bpb.de/apuz/223923/engagement-fuer-fluechtlinge?p=all> [Zugriff am 07.01.2019]

De Jong S. \& Ataç I. (2017), Demand and Deliver: Refugee Support Organizations in Austria. In: Social Inclusion, 5 (3), pp. 28-37.

Fioramonti L. \& Thümler E. (2013), Accountability, Democracy, and Post-growth: Civil Society Rethinking Political Economy and Finance. In: Journal of Civil Society, 9 (2), pp. 117128.

Fleischmann L. \& Steinhilper E. (2017), The Myth of Apolitical Volunteering for Refugees: German Welcome Culture and a New Dispositif of Helping. In: Social Inclusion, 5 (3), pp. $17-27$.

Hamann U. \& Karakayali S. (2016), Practicing Willkommenskultur: Migration and Solidarity in Germany. In: Intersections East European Journal of Society and Politics, 2 (4), pp. 69-86.

Han-Broich, M. (2012), Ehrenamt und Integration. Die Bedeutung sozialen Engagements in der (Flüchtlings-)Sozialarbeit. Wiesbaden, Springer VS.

Hannerz U. (1996), Transnational Connections - Culture, People, Places. London, Routledge.

Integrationsstelle \& Grundversorgungsstelle OÖ (2017), OÖ Integrationsbericht. URL: $<$ http://www.integrationsstelle-ooe.at/Mediendateien/RA_INTEGRATIONSBERICHT. pdf $>$ [Zugriff am 07.01.2019]

Janowicz C., Klement C. \& Mutz G. (2000), Corporate Volunteering neue Formen bürgerschaftlichen Engagements. In: Berliner Debatte, 11. Jg. Heft 4, pp. 17-26.

Karakayali S. \& Kleist O. (2016), EFA-Studie 2. Strukturen und Motive der ehrenamtlichen Flüchtlingsarbeit (EFA) in Deutschland. Berlin, Berliner Institut für empirische Integrationsund Migrationsforschung (BIM). Humboldt-Universität zu Berlin.

KAPELLER L. (2018), Mehr Integration und weniger Unterstützung, das geht nicht. Kurier.at URL: $\quad<$ https://kurier.at/politik/inland/mehr-integration-und-weniger-unterstuetzung-dasgeht-nicht/400008387> [Zugriff am 15.01.2019]

KÜHNLEIN I. \& BöHLE F. (2002), Motive und Motivationswandel des bürgerschaftlichen Engagements. In: Enquete-Kommission "Zukunft des Bürgerschaftichen Engagements" Deutscher Bundestag (ed.), Bürgerschaftliches Engagement und Erwerbsarbeit. Opladen, Leske u. Budrich, 267-297.

LAND OÖ (2008), Einbeziehen statt Einordnen. Zusammenleben in Oberösterreich. Integrationsleitbild des Landes OÖ. URL: <https://www.land-oberoesterreich.gv.at/files/publika tionen/So_Integrationsleitbild.pdf> [Zugriff am 07.01.2019] 
Langthaler H. \& Trauner H. (2009), Das österreichische Asylregime unter besonderer Berücksichtigung der Rolle zivilgesellschaftlicher Organisationen. In: SWS-Rundschau, 49 (4), pp. 446-467.

OÖ INTEGRATIONSRESSORT (2017), Masterplan Integration.

URL: <https://www.integrationsstelle-ooe.at/Mediendateien/MASTERPLAN_INTEGRA TION\%20O\%C3\%96.pdf $>$ [Zugriff am 07.01.2019]

Pries L. (2018), Introduction: Civil Society and Volunteering in the So-Called Refugee Crisis of 2015 Ambiguities and Structural Tensions. In: Feischmidt M., Pries L. \& Cantat C. (eds.), Refugee Protection and Civil Society in Europe. Cham: Palgrave Macmillan, pp. 1-23.

REINERT A. (2005), Bürgerschaftliches Engagement und demographischer Wandel. In: Bertelsmann Stiftung (ed.), Demographie konkret - Handlungsansätze für die kommunale Praxis. Aktion demographischer Wandel, pp. 24-33.

Rudolph B. (2003), Bürgerschaftliches Engagement im Wandel: Perspektiven für ein neues Geschlechtermodell? In: Feministische Studien, 2003 (01), pp. 123-136.

SCHEIBELHofer P. (2017), 'It won't work without ugly pictures': images of othered masculinities and the legitimisation of restrictive refugee-politics in Austria. In: NORMA - International Journal for Masculinity Studies, 12 (2), pp. 96-111.

Sen A. (1999), Ökonomie für den Menschen. Wege zu Gerechtigkeit und Solidarität in der Marktwirtschaft. München, Carl Hanser.

Simsa R. (et al.) (2016), Beiträge der Zivilgesellschaft zur Bewältigung der Flüchtlingskrise Leistungen und Lernchancen. Wien, Wirtschaftsuniversität Wien.

Simsa R. (2017), Leaving Emergency Management in the Refugee Crisis to Civil Society? The Case of Austria. In: Journal of Applied Security Research, 12 (1), pp. 78-95.

TÄUBIG V. (2009), Totale Institution Asyl: empirische Befunde zu alltäglichen Lebensführungen in der organisierten Desintegration. Weinheim, Juventa.

Vandevoordt R. \& Verschraegen G. (2018), Subversive Humanitarianism and Its Challenges: Notes on the Political Ambiguities of Civil Refugee Support. In Feischmidt M., Pries L. \& Cantat C. (eds.), Refugee Protection and Civil Society in Europe. Cham, Palgrave Macmillan, pp. 101-128.

Youkhana E. \& Sutter O. (2017), Perspectives on the European Border Regime: Mobilization, Contestation and the Role of Civil Society. In: Social Inclusion 5 (3), pp. 1-6.

Zimmer A. \& Vilain M. (2005), Bürgerschaftliches Engagement heute. Schriftenreihe der Stiftung Westfalen-Initiative, Bd.10. Ibbenbüren, Vereinsdruckerei GmbH.

\subsection{Internetquellen}

GemeIndehomepage St. Andrä-Wördern: <https://www.staw.at/Unsere_Gemeinde/Unser_Ort/ Zahlen_Fakten $>$ [Zugriff am 03. 12.2018].

STATISTIK AUSTRIA 2018: <http://www.statistik.at/wcm/idc/idcplg?IdcService=GET_PDF_FIL E\&RevisionSelectionMethod $=$ LatestReleased\&dDocName $=022914>$ [Zugriff ${ }^{-}$am 10. 01.2019].

UNDOK 2018: <http://arbeitsmarktzugang.undok.at/2013/02/06/faq-4/> [Zugriff am 12. 01.2019].

GemeindestatistiK NiederösterReich: <http://www.noe.gv.at/noe/St-Poelten/Gemeinden_im_ Bezirk_St._Poelten.html> [Zugriff am 08.01.2019]. 


\subsection{Interviews}

Interview mit Astrid, einer Ehrenamtlichen aus Gemeinde A, 15.11.2018, Sandra PunZ Gruppeninterview mit drei Senior/inn/en-Lehrer/inne/n aus Gemeinde B, 24.10.2018, Sandra PunZ

Interview mit Freiwillige/r/m Flüchtlingsinitiative Südburgenland, 16.05.2018, Hannah LICHTENWAGNER

Interview mit Regionalbetreuer/in, 16.05.2018, Hannah LiCHTENWAGNER

Interview mit Freiwillige/r/m Verein SOL, 29.10.2018, Hannah LichTENWAGNER

Interview mit Bildungsberater/in, 08.06.2018, Hannah LiCHTENWAGNER

Interview mit dem Obmann des Vereins ,grenzenlos“ am 05.05.2017, Andreas SchwarzBAUER

Interview mit einer ehrenamtlichen Deutschlehrerin am 23.05.2018, Andreas SchwarzBAUER

Interview mit dem Bezirkshauptmann eines oberösterreichischen Bezirkes, 26.07.2017, Sonja NeCHANKSY

Interview mit dem Bereichsleiter der Regionalen Kompetenzzentren für Integration und Diversität (ReKI), Volkshilfe OÖ am 10.09.2018, Sonja NECHANSKY

Interview mit Landesrat Anschober am 16.10.2018, Sonja NECHANSKY 